\title{
First Catalogue of Optically Variable Sources Observed by OMC Onboard INTEGRAL
}

\author{
Julia Alfonso-Garzón, Albert Domingo, and José Miguel Mas-Hesse \\ Departamento de Astrofísica, Centro de Astrobiología (INTA-CSIC) \\ POB 78, 28691 Villanueva de la Cañada, Spain \\ email: julia@cab.inta-csic.es
}

\begin{abstract}
In this work, we present the first catalogue of optically variable sources observed by the Optical Monitoring Camera (OMC), with information about the variability of more than 5000 objects and periodicity of $\sim 1000$ sources.
\end{abstract}

Keywords. catalogs, methods: data analysis, (stars:) binaries: general, (stars: variables:).

\section{Introduction}

The INTEGRAL Optical Monitoring Camera, OMC Mas-Hesse et al. 2003), observes the optical emission from the prime targets of the gamma ray instruments on-board the ESA mission INTEGRAL: SPI (gamma ray spectrometer) and IBIS (gamma ray imager), with the support of the JEM-X monitor in the X-ray domain. OMC provides photometry in the Johnson $\mathrm{V}$ band (centred at $5500 \AA$ ) and it is able to monitor sources from $\mathrm{V} \simeq 7 \mathrm{mag}$ (for brighter sources saturation effects appear) to $\mathrm{V} \simeq 16-17 \mathrm{mag}$ (magnitude limit for $3 \sigma$ source detection). Typical observations are done performing a sequence of different integration times, allowing for photometric uncertainties below 0.1 magnitude for objects with $\mathrm{V} \leqslant 16$. At this moment, the OMC database Gutierrez et al. 2004) contains light curves for more than 60000 sources (with more than 50 photometric points each).

\section{Data Analysis}

Selection of the sources Sources with more than 300 photometric points have been selected from the OMC database. In order to include only high-quality data, some selection criteria have been applied to individual photometric points, rejecting those ones that present saturation, low signal-to-noise, or are affected by cosmic rays.

Detection of variability We have fitted a constant to the data in the light curve (supposing the source is not variable). Then, we calculate the $\chi^{2}$ and the significance. This value gives the probability of being wrong when rejecting the null hypothesis (the source is constant). We have considered as variable those sources with $\alpha<0.05$ (probability of being variable of $95 \%$ ).

Study of the periodicity To determine which sources are periodic and to derive their periods, an algorithm based on the PDM technique (Phase Dispersion Minimization; Stellingwerf 1978) has been developed. This method divides the time-folded data into a series of bins and computes the variance of the amplitude within each bin with respect to a mean curve. This mean curve is obtained doing linear interpolations between the means of the bins. The ratio between the sum of the bin variances and the overall variance of the data set is called $\Theta$, and the period that minimizes this value will be the best estimate (see Fig. 1). Once this have been done, a visual inspection is needed to determine if the 
period is good enough. To calculate the error of the period, we have fitted a parabola to the peak of the periodogram corresponding to the minima. We have estimated the error as the distance from the $\mathrm{x}$-value at minima to the $\mathrm{x}$-value corresponding to a height in $\Theta$ equal to the deviation of the fit (see dashed orange lines in Fig. 1).

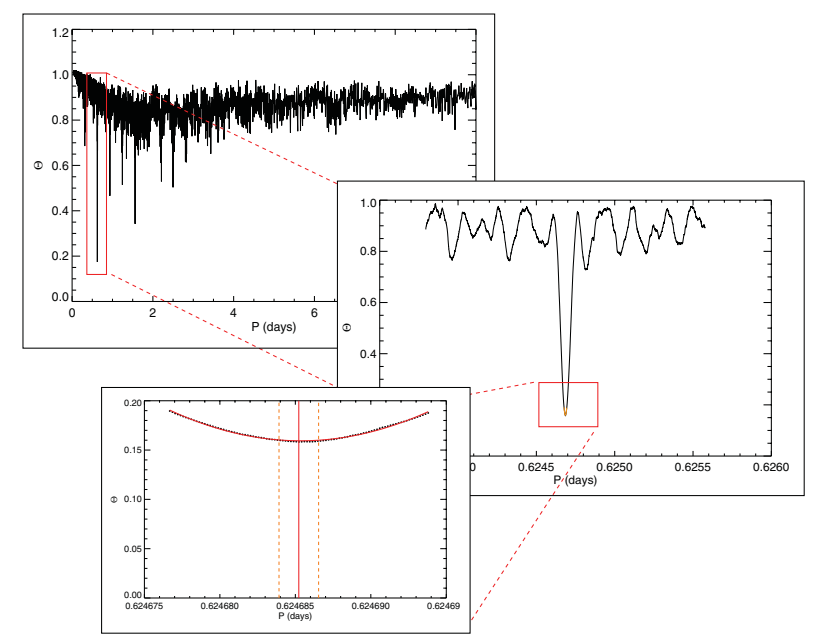

Figure 1. Visual description of the process of period determination for IOMC 0460000022 . Top: Periodogram from the full process. Middle: Zoom in the peak of the periodogram. Bottom: Detail of the parabolic fit in the peak of the minima used to determine the error of the period.

\section{Some results}

This first catalogue of optically variable sources observed by the OMC provides information about the variability of 5518 sources. When possible, we have studied the periodicity too, so we have determined good periods for approximately 1000 sources. The distribution of typical periods and object types can be found in Fig. 2. We have computed periods for several objects whose periodicity was unknown and, in many other cases, we have improved the results with respect to those found in the literature.
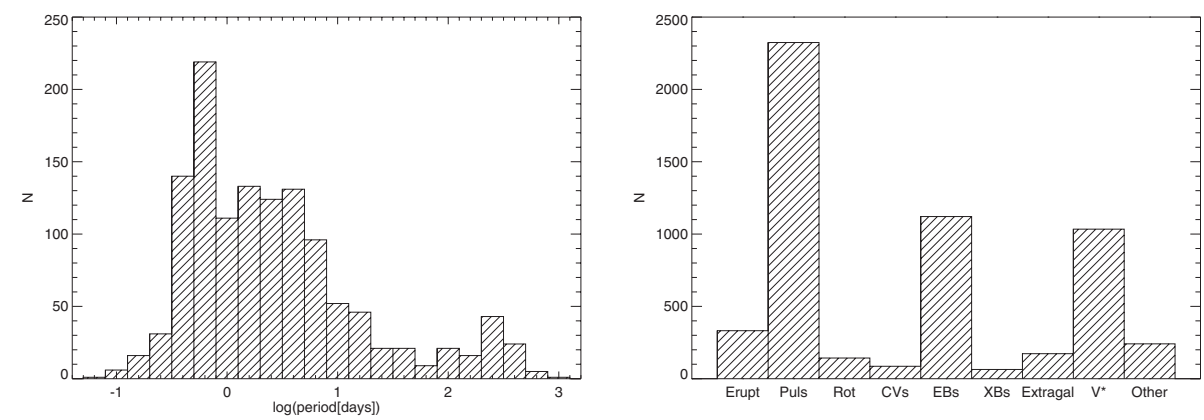

Figure 2. Left: Histogram of the periods derived. Typical values vary between a few hours and 10 days, with a peak of frequency in 15 hours. Right: Histogram of the type of objects identified in the catalogue. There are a big number of unclassified objects.

\section{References}

R. Gutiérrez, E. Solano, A. Domingo, \& J. García 2004, Astronomical Society of the Pacific Conference, Vol. 314, Astronomical Data Analysis Software and Systems XIII, 153

J. M. Mas-Hesse, A. Giménez, J. L. Culhane et al., 2003, A\&SA, 411, L261

R. F. Stellingwerf 1978, ApJ, 224, 953 